\title{
Una casa Manteña puede esconder otra: evaluación preliminar de la tola J6 de Japoto (provincia de Manabí, Ecuador)
}

Une maison Manteña peut en cacher une autre : première évaluation du monticule «J6 » de Japoto, province de Manabí, Équateur

A Manteño house may hide another: preliminary evaluation of the Japoto's J6 moundbuilding (Manabi province, Ecuador)

\section{Anne Touchard}

\section{OpenEdition}

Journals

Edición electrónica

URL: http://journals.openedition.org/bifea/3648

DOI: $10.4000 /$ bifea.3648

ISSN: 2076-5827

Editor

Institut Français d'Études Andines

Edición impresa

Fecha de publicación: 1 diciembre 2006

Paginación: 285-298

ISSN: 0303-7495

Referencia electrónica

Anne Touchard, «Una casa Manteña puede esconder otra: evaluación preliminar de la tola J6 de Japoto (provincia de Manabí, Ecuador) », Bulletin de l'Institut français d'études andines [En línea], 35 (3) | 2006, Publicado el 01 junio 2007, consultado el 02 diciembre 2020. URL : http:// journals.openedition.org/bifea/3648; DOI : https://doi.org/10.4000/bifea.3648

\section{(c) $(1)$}

Les contenus du Bulletin de l'Institut français d'études andines sont mis à disposition selon les termes de la licence Creative Commons Attribution - Pas d'Utilisation Commerciale - Pas de Modification 4.0 International. 


\section{Un sistema de producción artesanal de cuentas de concha en un contexto doméstico manteño: Japoto (provincia de Manabí, Ecuador)}

Mercedes Guinea*

\section{Resumen}

Los habitantes del actual Ecuador en el milenio anterior a la llegada de los españoles producían enormes cantidades de cuentas de concha para abastecer las demandas de sus elites y las de sus más potentes vecinos del sur. El análisis e interpretación de varias acumulaciones de preformas de cuentas de conchas localizadas en el montículo J4 del yacimiento de Japoto, un poblado manteño del Periodo de Integración (800-1535 d.C.), nos permite plantear algunas hipótesis acerca del sistema de producción en el que se enmarcaba la fabricación de dichas cuentas.

Palabras clave: arqueología, Ecuador, artesanía doméstica, cuentas de concha

\section{Un système de production artisanale de rondelles en coquille dans un contexte domestique manteño : Japoto (province de Manabí, Équateur)}

\section{Résumé}

Les hanciens habitants de l'Équateur ont fabriqué pendant le millénaire précédant la Conquête espagnole d'énormes quantités de rondelles taillées dans des coquilles de mollusques afin de répondre aux besoins en ornements de leurs élites et de leurs puissants voisins plus au Sud. L'analyse et I'interprétation de plusieurs accumulations de «pré-formes» de telles rondelles en coquille découvertes dans la tola J4 du site de Japoto (site de la période d'Intégration 800-1535 ap. JC) permet de proposer quelques hypothèses sur le système de production dont faisait partie la fabrication de ces rondelles.

Mots clés : archéologie, Équateur, artisanat domestique, rondelles de coquilles 


\title{
A system for handcrafting shell beads in a manteño domestic context: Japoto (Manabi province, Ecuador)
}

\begin{abstract}
In the millennium preceding the arrival of the Spanish, the inhabitants of today's Ecuador produced enormous quantities of shell beads to meet the demands of their elites and those of their more powerful neighbors to the south. Based on an analysis and interpretation of several accumulations of shell bead preforms found in Earthmound J4 at Japotó, an archaeological habitation site of the Integration Period (AD 800-1535), we discuss several hypotheses concerning the production system that resulted in these beads.
\end{abstract}

Key words: archaeology, Ecuador, domestic craft, shell beads

\section{INTRODUCCIÓN}

A pesar de que las cuentas de concha son hasta el momento el primer marcador disponible del advenimiento del pensamiento simbólico en el hombre moderno, hace más de 75000 años (d’Errico et al., 2005) y de que han desempeñado en todas la épocas y regiones del mundo una gran variedad de funciones, que van desde valor de intercambio a objetos rituales, no es mucho lo que se sabe acerca de los procesos tecnológicos de su fabricación en la América prehispánica. Este desconocimiento es especialmente destacable en el área septentrional andina, donde es prácticamente un lugar común entre los arqueólogos ecuatorianistas que los ManteñosHuancavilcas, durante el Periodo de Integración (800-1535 d.C.), producían y comerciaban grandes cantidades de cuentas de concha. Esta opinión está sustentada por las noticias de los cronistas y la masiva y constante aparición en las excavaciones de estos objetos. No obstante, la mayor parte de los trabajos especializados se centran en su valor como producto de intercambio a gran escala, especialmente las cuentas y valvas del preciado Spondylus, y poco se sabe de la naturaleza de la producción (Marcos, 1977; 1986; 1995; Murra, 1975; Norton, 1986; Paulsen, 1974). Esto se debe, entre otras cosas, al hecho de que generalmente la cuentas aparecen como un producto terminado que ofrece poca información sobre su proceso de fabricación. Afortunadamente algo está cambiando y en estos momentos ya tenemos, sin contar los de Japoto que presentamos aquí, datos de al menos otros siete sitios manteños con actividades relacionadas con la producción de cuentas de concha. El tipo de materiales, evidencias y circunstancias de su recuperación son muy diferentes en cada uno de ellos y todavía es pronto para generalizaciones aunque se observa una cierta evolución en los procesos de fabricación. En el yacimiento de Los Frailes (Mester, 1990; 1992), con raíces en la fase Guangala, inmediatamente anterior a la Manteña, la materia prima predominante es la Pinctada mazatlantica, aunque el Spondylus alcanza ya el $45 \%$ de la producción, que es poco especializada y tiene lugar en pequeños poblados. En el sitio manteño temprano más característico, Loma de los Cangrejitos (Marcos, 1981; Zevallos, 1995; Masucci, 2000; Carter, 1999, 2001a, 2001b; Marcos, 1981) la producción de cuentas de Spondylus es ya a gran escala y alcanza el $75 \%$ de la evidencia. La situación en López Viejo (Currie, 1995; 2001) es similar. Por el contrario, en los sitios manteños tardíos, que se sitúan en las inmediaciones de la playa, Salango 140 (Norton et al., 1983) Mar Bravo (Stothert \& Carter, 2000), Puerto Chanduy (Masucci, 2000), y Río Chico (Harris et al., 2004) se están capturando y procesando los Spondylus para la posterior fabricación de las cuentas en otros lugares. El estudio e interpretación de estos cambios observados forma parte de la tesis doctoral que está elaborando Ben Carter (comunicación personal). 
A pesar de pertenecer al periodo cronológico anterior, Guangala, y de ofrecer un tipo de evidencia muy distinto al nuestro, el Azúcar es otro sitio de especial interés en el área para el tema que nos ocupa. El trabajo de Maria Masucci (1995) nos brinda una información detallada de la tecnología de fabricación de las cuentas de conchas en el sitio y de la naturaleza de la producción. El Azúcar es un pequeño caserío, $25 \mathrm{~km}$ al interior, en donde, repartidas en 2,6 $\mathrm{m}^{2}$ de un basurero doméstico se recuperaron 617 cuentas, entre finalizadas y en proceso, junto con 6114 piezas de material de desecho y los instrumentos líticos de trabajo. No se ha establecido la duración de la acumulación, problema que es común a todos los anteriores talleres citados y que dificulta la comparación entre ellos y con el nuestro. Aunque hay algunas cuentas de Spondylus y Pinctada la mayor parte de la materia prima provenía de la Anadara tuberculosa y el Strombus gracilior. Para Masucci:

«la manufactura de las cuentas de conchas en El Azúcar es una actividad no especializada que está teniendo lugar en un contexto doméstico como una más de las actividades de subsistencia, para proveerse de un bien duradero intercambiable, en distintas situaciones sociales o de necesidad». (1995: 80-81)

Es en este contexto en el que hemos de analizar e interpretar las evidencias de manufactura de cuentas de concha localizadas durante la excavación del montículo J4 del yacimiento manteño de Japoto, en la costa de Manabí, que nos ofrecen unas cadenas operativas de fabricación no señaladas hasta el momento y nos permite plantear algunas hipótesis acerca del sistema de producción en el que estas se enmarcaban.

\section{EL SITIO DE JAPOTO}

El Periodo de Integración se caracteriza por un fuerte incremento de la complejidad sociopolítica en todo el territorio del actual Ecuador, pero muy especialmente en la zona costera en cuya mayor parte, durante este último periodo prehispánico, se asentaban grandes poblados pertenecientes a la cultura Manteña-Huancavilca, reconocibles arqueológicamente por grandes extensiones de montículos artificiales, conocidos familiarmente como tolas, la mayor parte de los cuales han sido destruidos por el crecimiento de la poblaciones actuales. El sitio de Japoto, citado por primera vez por Estrada (1957: 33; 1962: 28) como San Jacinto, es el único que se ha preservado en la costa de Manabí y es uno de los focos de atención del Proyecto Manabí Central desarrollado desde el año 2003 por la Misión Francesa en Ecuador en colaboración con la Universidad Complutense y el Cantón de Sucre. El yacimiento arqueológico, en las proximidades de la parroquia de San Jacinto, a 28 km de Bahía de Caráquez, se localiza a 2 km de la línea de la playa1. Los trabajos de prospección han localizado sesenta tolas, aunque todavía no se tiene la delimitación precisa del sitio debido a la vegetación impenetrable que lo recubre en parte. La intervención arqueológica durante los veranos de 2004, 2005 y 2006 se ha concentrado en el sector NE en el que las tolas visibles, con una altura entre 1 y $5 \mathrm{~m}$, tienen plantas rectangulares, ovaladas o redondas, alcanzando las más grandes hasta $60 \mathrm{~m}$ de largo, realizándose excavaciones en siete de ellas. Los materiales recuperados están en proceso de análisis, pero los primeros resultados de los estudios cerámicos confirman su pertenencia a la cultura manteña ${ }^{2}$. Hasta el momento solo tenemos una fecha de ${ }^{14} \mathrm{C}: 1100$ d.C.

2 Más información sobre el Proyecto Manabí y sus resultados en las página web: http://macgalatea.sip.ucm.es/web/ infoProyecto/manabicentral.php 


\section{1. Excavaciones en el montículo J4}

El montículo J4 se encuentra localizado en el sector noreste del sitio que, como hemos dicho, es el lugar en el que se centraron las excavaciones. Su altura sobre el estero cercano es de 3,5 m, es de forma ovalada y tiene una cima plana.

La primera intervención en el montículo se llevó a cabo en la temporada 20043. Se abrió una trinchera de 1,50 x 3,90 m orientada al eje principal, dividida en tres pozos de control (1, 2 y 3$)$. Aparentemente la primera ocupación se encontraba a 1,55 $\mathrm{m}$ de profundidad. Por encima de esta se observaron otras dos ocupaciones, todas con materiales manteños, separadas por niveles de relleno. En el suelo de la última de estas ocupaciones justamente al finalizar la capa de tierra vegetal se localizó el Rasgo 4 (Pozo 1), consistente en una gran acumulación de preformas de cuentas de concha, en distintos estadios dentro del proceso de fabricación (fig. 1a).
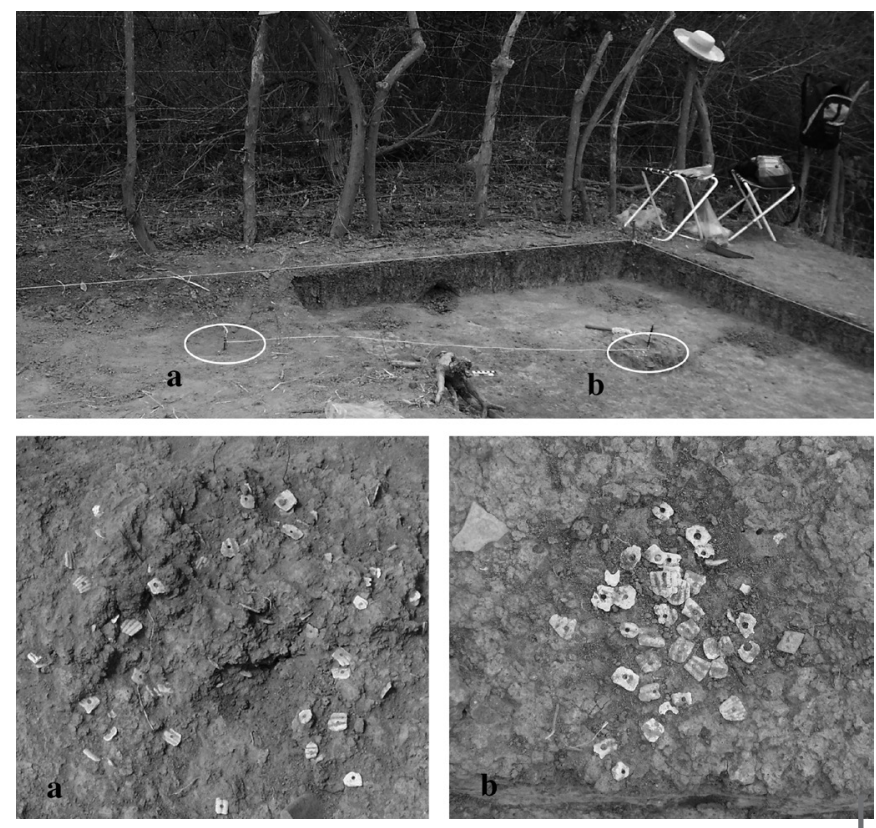

Figura 1 - Pozos 1 y K del montículo J4: localización y detalle de los Rasgos 4 (a) y 5 (b)

Foto M. Guinea
Con el fin de fundamentar algunas de las hipótesis preliminares de interpretación de este hallazgo, en la campaña de 2006 se procedió al decapado de toda el área accesible $(3,90 \times 8 \mathrm{~m})$ de la cima del montículo hasta alcanzar el suelo de la última ocupación, que se encontraba a una profundidad de $30 \mathrm{~cm}$ y sobre el último nivel de relleno de construcción. El resultado de esta nueva intervención fue la constatación de que el Rasgo 4 no era un elemento aislado, localizándose otro similar, el Rasgo 5 del Pozo K (fig. 1b), y otro gran número de preformas de cuentas (algunas prácticamente terminadas) en los pozos $L$ y $M$, de las cuales no ha sido posible saber si se depositaron agrupadas como las anteriores o no. Sobre el suelo se ubicaban también, dos huellas de poste (Rasgos 6 y 9)4, una pequeña plataforma de quincha (Rasgo 8)5, y los posibles restos de un festín depositados en un gran hoyo de poca profundidad (Rasgo 7)6, todo junto con objetos, restos de fauna, cerámica y otros, producto de la habitación continuada del lugar.

3 La excavación del montículo J4 ha sido llevada a cabo por María Ángeles Barriuso, arqueóloga del Proyecto Manabí.

4 Rasgo 6: huella de poste con ligera inclinación hacia sur de $30 \mathrm{~cm}$ de diámetro y $20 \mathrm{~cm}$ de profundidad, localizado en el extremo del suelo de actividad rellena de tierra suelta rojiza sin material. Rasgo 9: hueco de poste $(40 \mathrm{~cm}$ de diámetro y $15 \mathrm{~cm}$ de profundidad) relleno por pequeños fragmentos cerámicos.

5 Aunque técnicamente el término quincha se refiere a un sistema constructivo en el que las paredes de caña son revestidas con barro mezclado con paja y otros elementos. En Ecuador se hace un uso extensivo del término dando este nombre al barro preparado con la paja que se emplea en éste y otros muchos usos, entre otros para la preparación de suelos endurecidos con fuego. En este caso se ha construido con la quincha una plataforma de $10 \mathrm{~cm}$ de alto cubriendo un espacio de $110 \times 45 \mathrm{~cm}$ situada en el extremo del suelo de actividad con una posible función de separación o refuerzo.

6 Rasgo 7: hoyo $(1,50 \times 1,0)$ de 0,20 de profundidad conteniendo más de 2000 caracoles de agua dulce (Pomacea), restos de pescados grandes (cortes articulados), cerámica, piedras y otros restos alimenticios en una matriz de tierra ennegrecida y cenizas. La cantidad, calidad y concentración de estos restos nos permite plantear la hipótesis de que son los restos de un festín. 


\section{LOCALIZACIÓN ESPACIAL Y CARACTERÍSTICAS FÍSICAS DE LAS PREFORMAS DE CUENTAS DE CONCHA}

Dentro del yacimiento, el montículo que nos ocupa forma parte de un conjunto de tres montículos adosados: J3, el de mayor altura y extensión, J4 y J4B. Las evidencias observadas, que no es el caso detallar, nos permiten mantener la hipótesis de que las unidades J4 y J4B podrían ser áreas de actividad para los sucesivos ocupantes de las áreas de habitación localizadas en la J3. Sobre la superficie de la cima de la tola J4, en su última ocupación, una de estas actividades fue la fabricación de cuentas de concha. Como hemos dicho, en la mitad sur del área excavada (Pozos 1, 2, 3 y K), la evidencia de esta actividad se nos presenta agrupada en los Rasgos 4 y 5 (fig. 1a y b), mientras que en la mitad norte (Pozos $L$ y $M$ ) encontramos un gran número de preformas dispersas por toda la primera capa de ocupación (fig. 3).

\section{1. Los Rasgos 4 y 5}

El Rasgo 4 consistía en una acumulación de 1335 preformas de cuentas de concha, en distintos estadios dentro del proceso de fabricación (fig. 2a), cuya disposición en el terreno indicaba que se encontraban juntas dentro de un contenedor, bolsa, mate, cesto o similar, de material perecedero y forma redondeada. El $10 \%$ (131) de las preformas eran visibles en superficie, y se encontraban esparcidas por un área de $1 \mathrm{~m}$ de diámetro. A partir de ahí el radio de aparición iba disminuyendo, recogiéndose 81 (6\%) en los primeros $15 \mathrm{~cm}$ de excavación. Entre 23 y $30 \mathrm{~cm}$ de profundidad se apreció claramente en el terreno una mancha circular de $30 \mathrm{~cm}$ de radio, consistente en tierra gris, oscura y suelta posiblemente resultado de la descomposición del posible contenedor (fig. 1a). En estos $7 \mathrm{~cm}$ se agrupaban el grueso de las preformas, 1077 (80\%). En los siguientes $5 \mathrm{~cm}$ todavía se recuperaron $52(4 \%)$ preformas. De esto es posible deducir que las preformas se encontraban depositadas en el suelo dentro de un contenedor, que las mantenía agrupadas y que durante el proceso tafonómico este fue destruido aflorando a la superficie parte de las preformas por la acción de las raíces de las plantas, las lluvias y otros, manteniéndose in situ la mayor parte de ellas.

Solo se ha encontrado un útil que pudiera encontrarse en el contenedor junto con las preformas, una lámina de obsidiana, y aún esta con la salvedad de que pudiera ser

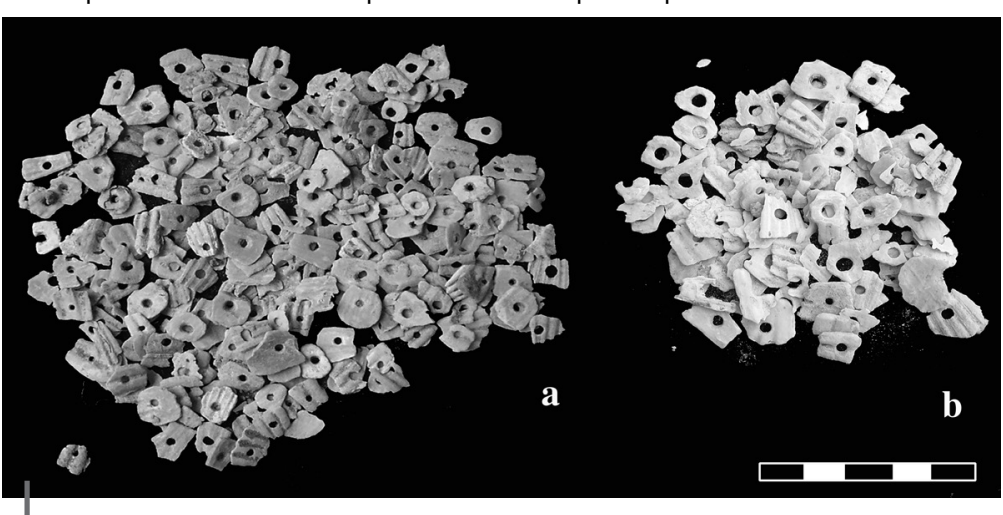

Figura 2 - Algunas de las preformas de cuentas que se agrupaban en los Rasgos 4 (a) y 5 (b)

Foto M. Guinea intrusiva dado que, como veremos más abajo, nada indica que fuera utilizada en el trabajo de las conchas. En distintos lugares del área excavada se han recuperado 2 lascas de pedernal, lo que pudiera ser un fragmento de yunque, tres alisadores y una plaquita de piedra fragmentada. Todos estos artefactos aparecen con relativa frecuencia en el yacimiento y con características tipológicas muy similares, por lo que de momento7 no es posible hacer una asociación directa de 

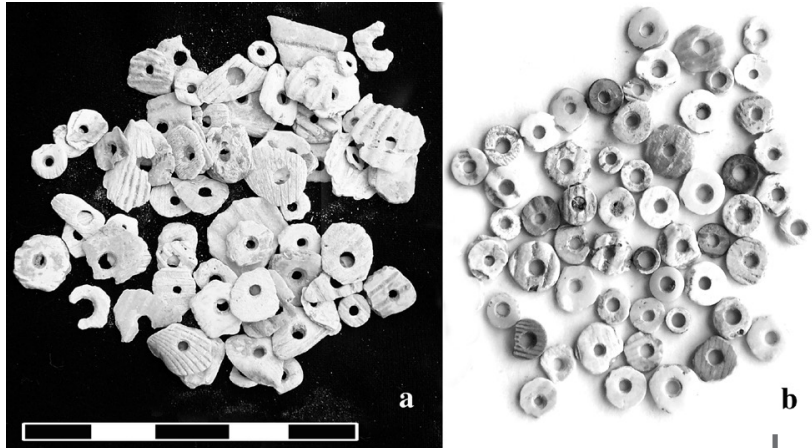

Figura 3 - Parte de las preformas que se localizaron dispersas en los pozos $\mathrm{L}$ y $\mathrm{M}$

a) De especimenes adultos de Argopecten

b) De especimenes juveniles y cuentas acabadas

Foto M. Guinea estos útiles con los trabajos de las conchas, aunque es muy posible. Es destacable la localización a medio metro del rasgo una vasija cerámica fragmentada grande, de paredes rectas y fondo plano, de un tipo no frecuente en el yacimiento.

El Rasgo 5 se encontraba muy próximo $(1,20 \mathrm{~m}$ al sur y $30 \mathrm{~cm}$ al oeste) del Rasgo 4 (fig. 1), y es similar a este, aunque no era visible en superficie apareciendo solo al retirar la capa vegetal. Consistía en una acumulación de 401 preformas de cuentas de concha -igualmente - en distintos estadios dentro del proceso de fabricación (fig. 2b) y de nuevo su disposición en el terreno indicaba que estas se encontraban juntas dentro de un contenedor de material perecedero y forma redondeada

de $30 \mathrm{~cm}$ de diámetro y $15 \mathrm{~cm}$ de alto (fig. 1b). El proceso tafonómico también removió parte del contenido y otras 129 preformas se encontraban siguiendo la trayectoria de una raíz cercana. Si sumamos a estas las 122 que iban aflorando hacia la superficie nos da un total de 652 preformas (incluyendo 184 fragmentos). En este caso sin duda, dentro del posible contenedor había también una piedra pequeña, dura y negra y asociados directamente se encontraban un tortero, una aguja de cobre, una empanadilla ${ }^{8}$ y una vasija cerámica fragmentada grande, de paredes rectas y fondo plano similar a la que se encontraba en la proximidades del rasgo anterior. En el resto del pozo se recuperaron un fragmento de yunque, un perforador de piedra, dos pulidores y una lasca de obsidiana y otra de pedernal.

La materia prima de los materiales de los Rasgos 4 y 5 es la concha de un pectínido, con toda probabilidad el Argopecten circularis (Sowerby, 1835) (Keen, 1971: 87) conocido en Ecuador como concha de abanico9. Este bivalvo alcanza en su madurez entre 45 y $50 \mathrm{~cm}$ y el tamaño y la separación de las costillas radiales, junto con la finas laminillas de crecimiento, que se observan en los fragmentos arqueológicos indica que estos corresponden a individuos adultos. Su uso como materia prima para la fabricación de cuentas de concha no está señalado en la arqueología del Ecuador, pero hay que tener en cuenta que una vez que las cuentas están terminadas es prácticamente imposible en la mayoría de los casos, quizás con la excepción del Spondylus, determinar la especie de origen, ya que han perdido su superficie por el pulido, e incluso el color por el paso de el tiempo. Los colores del Argopecten circularis van desde el blanco al naranja oscuro, pasando por el rosado y púrpura. La gran mayoría de las preformas de Japoto han perdido estos colores, como es habitual.

8 Porción de tierra comestible envuelta en una hoja, y sometida después a una ligera cocción u horneado, de las que se han recuperado 297 en el yacimiento de Japoto y 132 en la tola J4.

9 El Argopecten circularis es una especie marina que habita en aguas moderadamente profundas (entre 20 a 40 metros) y puede acomodarse sobre una gran variedad de sustratos. Esta especie puede ser encontrada desde Bahía Monterrey, California, hasta Paita, Perú. En el Ecuador actual se la ha encontrado en Esmeraldas y Jaramijó en la Provincia de Esmeraldas, en Manta, Puerto Cayo, Machalilla y Salango en la Provincia de Manabí, y en la Punta de Santa Elena y Playas en la Provincia del Guayas (Mora, 1990). 


\section{2. Los pozos L y M}

En estos pozos encontramos 933 preformas dispersas (fig. 3) mezcladas con la matriz de tierra de la ocupación en vez de agrupadas como en el apartado anterior. No ha sido posible determinar si esta diferencia se debe, en su totalidad o en parte, a un distinto proceso tafonómico o al desarrollo de la propia actividad de fabricación de cuentas, aunque personalmente me inclino por lo primero, ya que la superficie de la tola ha sido cultivada frecuentemente y lo que es realmente una gran suerte es que se hayan conservado los Rasgos 4 y 5 . Una diferencia más significativa es que aquí junto a las preformas encontramos también el producto final: 195 cuentas circulares discoidales terminadas o a punto de serlo (fig. $3 b$ ), ya que se podría interpretar como que en esta área se llevaba a cabo la última fase del proceso de fabricación. Otra diferencia observable es que en su mayor parte el tamaño y la separación de las costillas radiales de las valvas del Argopecten circularis corresponden a las medidas de individuos juveniles (fig. 3b), aunque también están presentes los adultos (fig. 3a). En algunos casos la materia prima pudiera ser un especie distinta de pectínido u otro bivalvo no identificado. En estos pozos se encontró una cantidad de útiles de piedra algo mayor que en los otros, entre ellos 11 lascas de obsidiana, 6 de pedernal, núcleos de ambos materiales y 2 fragmentos de yunque.

\section{3. Las preformas}

Las 2920 preformas pueden agruparse atendiendo a su perímetro en seis tipos: irregulares, triangulares, rectangulares, trapezoidales, cuadradas y redondeadas (fig. 4). Dentro de cada una de ellas la estandarización de sus dimensiones nos indica la intencionalidad de su fabricación (cuadro 1) y como veremos más abajo se corresponde con un estadio dentro de la cadena operativa 10 .

Todas están perforadas y el diámetro del orificio $(0,26 \mathrm{~cm})$ es la más regular de las medidas (cuadro 1$)$. La perforación es en general cónica, se efectúa desde la superficie interior de la valva (fig. 5a), en la mayor parte aprovechando el menor espesor de la zona intercostillar (fig. 4d), y muestra un alto grado de homogeneidad y estandarización. A veces se retoca ligeramente por el otro lado. Al menos en las ocho piezas que han sido observadas por Microscopía Electrónica de Barrido esta operación parece haber sido realizada con una lasca de pedernal ${ }^{11}$.

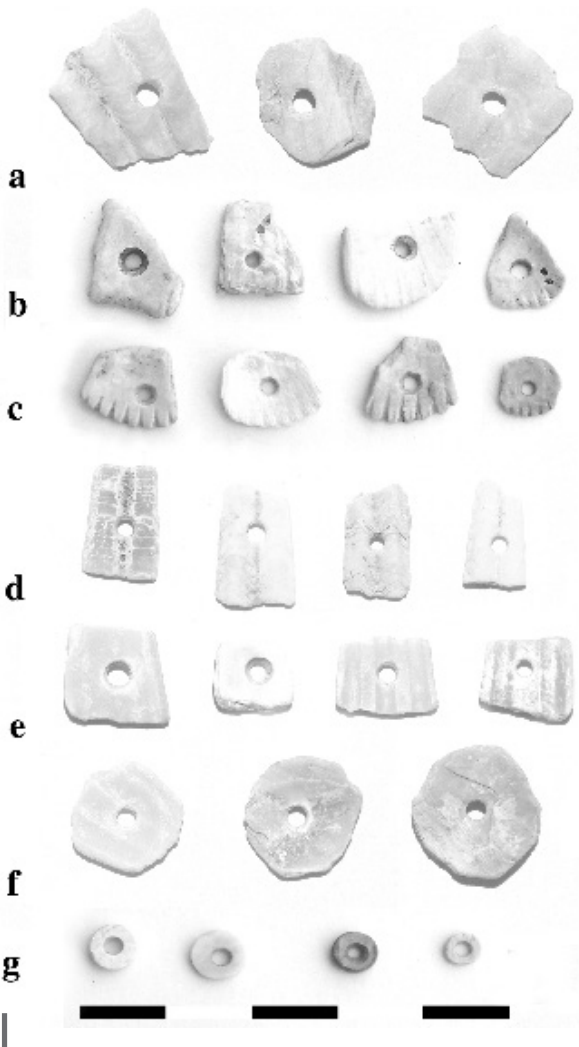

Figura 4 - Tipos de preformas en el montículo J4 a) irregulares, b) triangulares, c) trapezoidales, d) rectangulares, e) cuadradas, f) redondeadas y g) terminadas Foto M. Guinea

10 Aunque para el establecimiento de las cadenas operativas se ha empleado la colección completa, el análisis estadístico se ha efectuado sobre una muestra de 947 piezas (30\%).

11 La caracterización de las de huellas de manufactura de estas ocho piezas ha sido llevada a cabo por Emiliano Melgar Tisoc y Reyna Beatriz Solís Ciriaco en el Laboratorio de Microscopía Electrónica de la Subdirección de Laboratorios y Apoyo Académico del Instituto Nacional de Antropología e Historia de la ciudad de México. Utilizando un Microscopio Electrónico de Barrido JEOL (100x,300x, 600x y 1000x) JSM-6460LV, en modo de alto vacío y empleando los mismos parámetros de distancia (10mm), haz (49), voltaje (20kV) y señal (SEI). 
Cuadro 1 - Valores medios de las características físicas de las preformas y cuentas de concha del montículo J4 de Japoto, en conjunto y por unidades

\begin{tabular}{|c|c|c|c|c|c|c|c|c|c|}
\hline \multirow[t]{2}{*}{$\begin{array}{c}\text { J4 TOTAL } \\
\text { (2920 Preformas y } \\
195 \text { cuentas) }\end{array}$} & \multicolumn{2}{|c|}{$\begin{array}{l}\text { LARGO } \\
(\mathrm{cm})\end{array}$} & \multicolumn{2}{|c|}{$\begin{array}{l}\text { ANCHO } \\
(\mathrm{cm})\end{array}$} & \multicolumn{2}{|c|}{$\begin{array}{l}\text { ESPESOR } \\
(\mathrm{cm})\end{array}$} & \multicolumn{3}{|c|}{$\begin{array}{c}\text { PERFORACIÓN } \\
\text { MÁXIMA } \\
(\mathrm{cm})\end{array}$} \\
\hline & $\mathrm{m}$ & $\mathrm{dt}$ & $\mathrm{m}$ & $\mathrm{dt}$ & $\mathrm{m}$ & $\mathrm{dt}$ & $\mathrm{m}$ & $\mathrm{dt}$ & rango \\
\hline Irregulares & 1,24 & 0,18 & 1,20 & 0,21 & 0,17 & 0,04 & 0,29 & 0,05 & $0,10-0,50$ \\
\hline Triangulares & 1,02 & 0,16 & 0,87 & 0,14 & 0,15 & 0,04 & 0,25 & 0,06 & $0,10-0,50$ \\
\hline Trapezoidales & 0,90 & 0,15 & 0,77 & 0,11 & 0,14 & 0,03 & 0,22 & 0,04 & $0,10-0,40$ \\
\hline Rectangulares & 1,04 & 0,25 & 1,11 & 0,19 & 0,17 & 0,04 & 0,27 & 0,05 & $0,10-0,50$ \\
\hline Cuadradas & 0,90 & 0,15 & 0,98 & & 0,16 & 0,04 & 0,25 & 0,05 & $0,10-0,40$ \\
\hline Redondas & 0,90 & 0,20 & 0,90 & 0,21 & 0,16 & 0,05 & 0,25 & 0,05 & $0,10-0,45$ \\
\hline $\begin{array}{l}\text { Total mue } \\
\text { (890 pref }\end{array}$ & 0,98 & 0,20 & 0,98 & 0,23 & 0,16 & 0,04 & 0,26 & 0,05 & $0,10-0,50$ \\
\hline $\begin{array}{l}\text { Terminadas } \\
\text { (82 cuentas) }\end{array}$ & 0,49 & 0,06 & 0,48 & 0,06 & 0,12 & 0,03 & 0,17 & 0,04 & $0,10-0,30$ \\
\hline
\end{tabular}

\section{J4 UNIDADES}

\begin{tabular}{|c|c|c|c|c|c|c|c|c|c|}
\hline $\begin{array}{l}\text { Rasgo } 4 \\
\text { (1335 preformas) }\end{array}$ & 1,03 & 0,18 & 1,18 & 0,17 & 0,21 & 0,06 & 0,28 & 0,05 & $0,10-0,50$ \\
\hline $\begin{array}{l}\text { Rasgo } 5 \\
\text { (652 preformas) }\end{array}$ & 1,16 & 0,18 & 0,99 & 0,14 & 0,19 & 0,05 & 0,29 & 0,05 & $0,10-0,50$ \\
\hline $\begin{array}{l}\text { Pozos L y M } \\
\text { (933 Preformas) }\end{array}$ & 0,83 & 0,19 & 0,67 & 0,15 & 0,14 & 0,04 & 0,22 & 0,04 & $0,10-0,50$ \\
\hline (195 Cuentas) & 0,49 & 0,06 & 0,48 & 0,06 & 0,12 & 0,03 & 0,17 & 0,04 & $0,10-0,30$ \\
\hline
\end{tabular}

Aún las preformas de perímetro irregular (fig. 4a) muestran una cierta regularidad en su tamaño, $1,24 \times 1,20 \mathrm{~cm}$, muy próximo al tamaño medio de las preformas redondas las más acabadas, $0,90 \mathrm{~cm}$ de diámetro, lo que nos indica su selección (cuadro 1). Todas, incluidas las primeras (fig. 5c), presentan huellas de desgaste superficial y distintos estados de acabado (fig. 5b), realizados con una piedra aún no identificada12. El espesor medio es $0,16 \mathrm{~cm}$. El producto finalizado serían unas cuentas circulares discoidales (fig. 4g) de unas dimensiones medias de $0,49 \mathrm{~cm}$ de diámetro, un espesor de $12 \mathrm{~cm}$ y una perforación $17 \mathrm{~cm} 13$ representadas por las 195 recuperadas en los Pozos L y M (fig. 3b). Debido a las diferencias de materia prima citadas, las medias de las preformas de los pozos $\mathrm{L}$ y $\mathrm{M}$ son un poco más pequeñas que las de los Rasgos 4 y 5, que a su vez presentan algunas diferencias entre sí, siendo en general un poco más grandes las preformas del segundo (cuadro 1).

12 Estamos esperando nuevos resultados, pero de momento, por el análisis de sus huellas mediante MEB sabemos que no es basalto, ni caliza, ni granito, ni obsidiana, ni pizarra.

13 La diferencia entre la perforación máxima media de las preformas y la de las cuentas terminadas es debida a que la perforación fue efectuada antes que el desgaste y por lo tanto en el primer caso tenemos la medida de la base del cono perforado y en el segundo la de su parte central. 


\section{LAS CADENAS OPERATIVAS DE FABRICACIÓN DE CUENTAS}

El análisis detenido de las piezas y sus huellas de manufactura nos han permitido reconstruir una cadena operativa de producción con dos variantes (fig. 6), destacando dentro de ellas la gran diversidad de sus modos de ejecución. En ambas variantes, las tres primeras etapas del proceso de elaboración de las cuentas de concha no están presentes en el área excavada, por lo que debieron realizarse en otro lugar por los mismos o distintos artesanos. $\mathrm{Ni}$ en la unidad J4, ni en el resto del yacimiento de Japoto se han localizado restos de talla o valvas enteras, que nos den una pista del lugar donde se efectuaba esta operación.

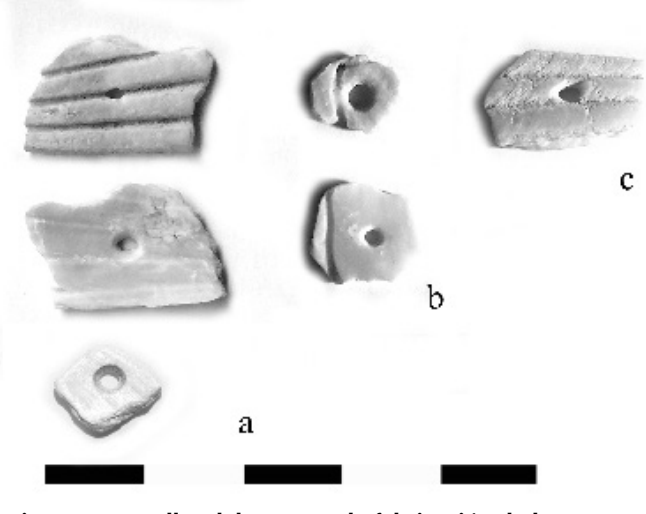

Figura 5 - Huellas del proceso de fabricación de las cuentas Foto M. Guinea

\section{1. Cadena operativa variante 1 (fig. 6a)}

- Selección de la materia prima: las valvas de especimenes adultos de Argopecten circularis.

- Fragmentación de las valvas enteras.

- Corte por percusión de los fragmentos obtenidos para regularizarlos. Estos fragmentos están todos ya perforados en todas las preformas, por lo que es solo la lógica lo que nos lleva a suponer que este estadio es anterior a la perforación.

- Perforación con una lasca de pedernal. Hay que hacer notar que esta operación no se lleva a cabo en un único momento, de modo que tenemos distintos estados de acabado del orificio de la cuenta en todos los tipos de preformas, repartidos de forma irregular.

- Corte por percusión, en el sentido las costillas radiales para obtener unas preformas rectangulares.

- Recorte perpendicular al anterior para obtener unas preformas cuadradas.

- Retalla para redondear las anteriores.
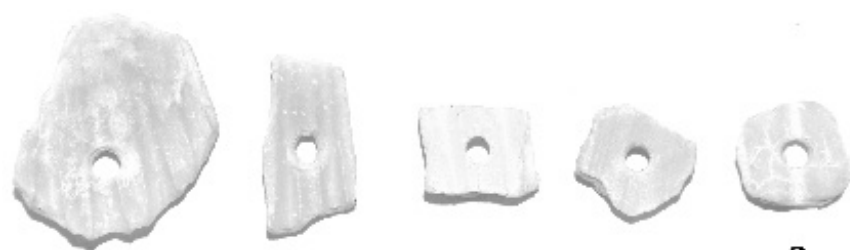

$\mathbf{a}$
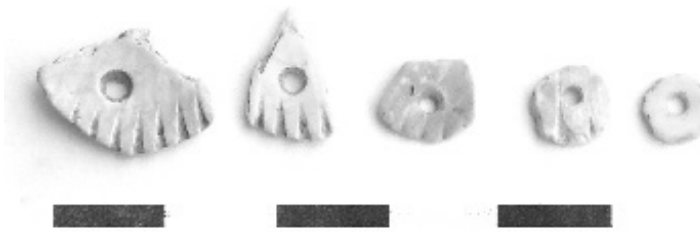

b

Figura 6 - Cadena operativa de la fabricación de las cuentas de concha en el montículo J4: a) variante 1 , b) variante 2

Foto M. Guinea 
- Desgaste, pulido y/o bruñido con una piedra no identificada. Lo más destacable de esta actividad es que, aún en mayor medida que la perforación, no parece realizarse en un momento preciso de la cadena operativa, y tenemos evidencias de esta tarea en todos los estadios desde el número 3.

- Ensartado en un hilo para su desgaste y pulido final.

\section{2. Cadena operativa variante 2 (fig. 6 b)}

- Selección de la materia prima: valvas especimenes juveniles y adultos Argopecten circularis y posiblemente un especie distinta de pectínido u otro bivalvo no identificado.

- Fragmentación de las valvas enteras.

- Corte por percusión de los fragmentos obtenidos para regularizarlos. Igualmente estos fragmentos están todos ya perforados en todas las preformas.

- Perforación con una lasca de pedernal.

- Corte por percusión para obtener unas preformas triangulares.

- Corte de uno de los vértices del triángulo obteniendo unas preformas trapezoidales.

- Retalla para redondear las anteriores.

- Desgaste, pulido y/o bruñido con una piedra no identificada.

- Ensartado en un hilo para su desgaste y pulido final.

En el estado actual de la investigación pensamos que la diferencia entre las dos variantes se debe más a configuración de la preforma irregular de partida que a la idiosincrasia del operario, ya que ambas formas de trabajar se encuentran en los dos contenedores. Si bien, la primera predomina en el Rasgo 4, mientras que en el 5 y en los pozos L y M lo hace la segunda. Tampoco es posible asociar esta diferencia a la materia prima ya que aunque parece que todas las especies juveniles fueron trabajadas con la variante 2 de la cadena operativa, esta también se encuentra aplicada a los Argopecten adultos.

Hay dos puntos a destacar dentro de esta cadena operativa. El primero es que la perforación (estadio 4), muy homogénea y estandarizada, se llevaba a cabo sobre las preformas obtenidas de la fragmentación de las valvas (fig. 4a, 6) antes de cualquier labor de desgaste o acabado, cuando lo más común es perforar la preforma cuando ya está redondeada y con parte del acabado. El segundo es que no hay un orden fijo de ejecutar las operaciones de desgaste y acabado (fig. 5), ni de retoque de la perforación, lo que sugiere que el artesano las llevaba a cabo de forma arbitraria de acuerdo a sus intereses o circunstancias del momento. El contraste entre la homogeneización de la perforación, que sugiere un proceso de elaboración estandarizado, frente a la libertad de ejecución aparente del resto de las tareas de la cadena, junto al hecho de que no encontremos ninguna preforma sin perforar en el conjunto fundamenta la hipótesis de que pudieron realizarse por distintos artesanos, incluso, como explicaremos más adelante, en distintos lugares. Hay que señalar también, que en relación con otros sitios manteños, las cuentas de concha obtenidas en las distintas intervenciones en el sitio de Japoto son más bien escasas y ni siquiera se han encontrado como ajuar en ninguno de los entierros localizados.

Con anterioridad a nuestro hallazgo, Carter (2001a; b) identificó dos tipos de cadenas operativas empleadas por los manteños. La Cadena 1, utilizada principalmente en el Manteño Temprano, se realizaba en cinco etapas: percusión de la concha, desgaste de las dos caras planas, aproximación a la forma final, perforación con buril y desgaste y pulido final de la sarta de cuentas. La Cadena 2, típica de los sitios tardíos era más simple y solo tenía tres etapas de elaboración: recojo de fragmentos de concha en la playa y redondeo de la cuenta, desgaste de los bordes y perforación con un buril. Por su parte, la cadena operativa del taller guangala de El Azúcar, aunque la publicación no da muchos detalles, parece que podría incluirse en el 
tipo 1 de Carter, si bien Masucci (1995: 75) hace notar que las cuentas parecían haber sido perforadas antes y después de haber sido dadas forma y acabado, lo que las asemejaría a las nuestras. La cadena operativa que se evidencia en Japoto no concuerda con ninguna de las anteriormente propuestas.

\section{EL SISTEMA DE PRODUCCIÓN ARTESANAL DE JAPOTO}

Si bien, como hemos visto en la presentación, hay otros casos de contextos de producción doméstica de cuentas de concha en Ecuador y en otros lugares (El Azúcar), no conozco ningún caso con las características de los Rasgos 4 y 5: algún tipo de contenedor que se conservaba en el espacio doméstico, conteniendo un material en proceso que evidencia una cadena operativa original y una gran variabilidad en el orden de ejecución de las labores de acabado del producto. El referente interpretativo más similar que he encontrado es el sistema de producción de cuentas de los habitantes actuales del Kalahari, cuya tradición de ejecución se remonta a la Prehistoria, aunque la materia prima son las cáscaras de huevo de avestruz. La doctora Louis Fourie recogió en los años veinte del pasado siglo una serie de materiales y fotografías de mujeres Khoisan fabricando este tipo de cuentas, que se conservan en el museo de África de Johannesburgo. Una de estas fotos (Vanhaeren, 2003: 30, fig.1 abajo a la derecha) presenta una cadena operativa que coincide con el rasgo menos común de la de Japoto: la perforación se efectúa sobre las piezas irregulares, antes de darles forma.

No acaban aquí las felices coincidencias, uno de los materiales que se guardan en el museo es una bolsa donde la artesana guardaba 555 cuentas en diferentes estadios de manufactura, de las que más de la mitad son preformas parcialmente perforadas, un tercio cuentas perforadas totalmente pero no acabadas y el resto piezas irregulares, preformas redondeadas sin perforar y dos cuentas terminadas (Vanhaeren, 2003: 11-12). Las mujeres San llevan consigo este tipo de bolsas, trabajando en los materiales que contienen cuando tienen ocasión dentro de sus actividades domésticas, mientras conversan en pequeños y relajados grupos a la sombra de los árboles 14 .

En la sociedades San estas cuentas son objetos de intercambio que sirven para reforzar las redes sociales y las relaciones económicas (Wiessner, 1982). La analogía etnográfica con estos grupos ha sido utilizada con éxito en la interpretación del sistema y función de la producción de cuentas de los primeros homínidos africanos, cuyas evidencias arqueológicas eran similares, dentro de una tradición que parece permanecer sin variaciones a través del tiempo (Vanhaeren, 2003: 17). En nuestro caso también es posible aplicar esta analogía, por supuesto con toda clase de cautelas, a la interpretación del contexto de producción de nuestro hallazgo cuyas características se ajustan casi perfectamente a este modelo: una manufactura no especializada que se integra en la vida individual, se realiza en un entorno doméstico y cuyo material en proceso se conserva mezclado en un contenedor.

Un cuestión diferente es el papel de esta producción dentro de la sociedad manteña, mucho más compleja que la San. En relación a este tema hay dos puntos a tener en cuenta y que nos diferencian del ejemplo San: la materia prima, que en nuestro caso no está ampliamente distribuida por todo el territorio, sino que se concentra en los pueblos costeros y la falta de los dos primeros estadios de la cadena operativa, selección y fragmentación de las valvas, que puede interpretarse como que éstos fueron realizados en otros lugares. Si aceptamos esta hipótesis de trabajo, habremos encontrado la única evidencia hasta el momento de la segunda parte de la cadena operativa del Manteño Tardío, cuya primera parte se lleva a cabo en los grandes talleres de captura de los moluscos y selección y fragmentación de las conchas (Salango, Mar Bravo, Puerto Chanduy, Río Chico), desde donde se distribuían a otros lugares no especializados que fabricaban las cuentas, uno de los cuales pudo ser Japoto.

14 Se puede observar esto en las fotos actuales tomadas por I. DeVore que se encuentran en el archivo virtual de Anthro-Photo File en http://www.discoverlife.org/ap/. Archivos : APDEV_0813, 0814, 0816, 0784, 1044. 
En el estado actual de la investigación sobre el tema, es posible proponer una evolución en la manera en que se está llevando a cabo esta tarea dentro de la sociedad manteña. En un primer momento, continuando con la tradición Guangala (El Azúcar) hay una producción doméstica como una más de las actividades de subsistencia (Los Frailes), más tarde y coincidiendo con un aumento de la demanda, especialmente de sus vecinos de más al sur, la producción aumenta su escala y se focaliza en el Spondylus que es la especie más solicitada (La Loma de los Cangrejitos, López Viejo). En el Manteño Tardío, hay una diversificación de la producción, que se integra en una red en la cual existen por un lado, talleres de captura y procesamiento de los Spondylus y otras especies valiosas como los pectínidos (Salango, Mar Bravo, Puerto Chanduy, Río Chico) y por otro, contextos domésticos (Japoto) en los que se elaboran las cuentas, presumiblemente puestos en contacto a través de grupos de comerciantes o mercados 15 .

\section{Referencias citadas}

CARTER, B. P., 1999 - Beads of Change: An Experimental Approach to Shell Beads Production on the Santa Elena Peninsula, Ecuador; New Orleans. Paper Presented at the 64th Meeting of the Society for American Archaeology.

CARTER, B. P., 2001a - Informe preliminar sobre el estudio de las Cuentas de Concha de Loma de los Cangrejitos, Ecuador; Guayaquil. Ms. en el Instituto Nacional de Patrimonio Cultural.

CARTER, B. P., 2001b - Microliths and Microbeads: Preliminary Análisis of Shell Vedas From Loma de los Cangrejitos, Shouthwestern Ecuador, Chicago. Posters Presented at the 66th Meeting of the Society for American Archaeology.

CURRIE, E. J., 1995 - Prehistory of the South Manabi Coast, Ecuador-Lopez Viejo, 64 p.; Oxford: BAR International Series. Tempus Reparatum 618.

CURRIE, E. J., 2001 - Manteño, Ceremony and Symbolism: Mortuary Practices and Ritual Activities at Lopez Viejo, Manabi, Ecuador. In: Mortuary Practices and Ritual Associations: Shamanistic Elements in Prehistoric Funerary Contexts in South America (Currie \& Staller, eds.): 67-92; Oxford: BAR International Series, Archaeopress, 982.

D'ERRICO, F., HENSHILWOOD, C., VANHAEREN, M. \& van NIEKERK. K., 2005 - Nassarius kraussianus shell beads from Blombos Cave: Evidence for symbolic behaviour in the Middle Stone Age. Journal of Human Evolution, 48: 3-24; Academic Press.

ESTRADA, E., 1957 - Prehistoria de Manabí, 82 p.; Guayaquil: Publicación del Museo Víctor Emilio Estrada 3.

ESTRADA, E., 1962 - Arqueología de Manabí Central, 205 p.; Guayaquil: Publicación del Museo Víctor Emilio Estrada 7.

HARRIS, M., MARTÍNEZ, V., KENNEDY W. M., J., ROBERTS, C. \& GAMMACK-CLARK, J., 2004 - The Complex Interplay of Culture and Nature in Coastal South-Central Ecuador: An Interdisciplinary Work. Expedition, 46: 38-43; Philadelphia: University of Pennsylvania Museum.

15 Esta investigación ha sido parcialmente financiada por el Ministerio Español de Educación y Ciencia (TIN200508788-C04-04). Agradecemos a Emiliano Melgar su desinteresada ayuda con el análisis de las huellas de fabricación de las cuentas y María Ángeles Barriuso el meticuloso proceso de excavación de los hallazgos. 
Un sistema de producción artesanal de cuentas de concha en un contexto doméstico manteño

KEEN, M., 1971 - Seashells of Tropical West America: Marina Molluscs From Baja California to Peru, 1064 p.; Stanford: University of Stanford Press.

MARCOS, J. G., 1977 - Cruising to Acapulco and Back With the Thorny Oyster set: A Model for a Lineal Exchange System. Journal of Steward Anthropological Society, 9 (1-2): 99-132; Illinois: Urbana.

MARCOS, J. G.,1981 - Informe sobre el área ceremonial del complejo Manteño Huancavilca de La Loma de Los Cangrejitos, Valle de Chanduy, Ecuador (Ogsech 4). El Arquitecto, 1 (5): 54-63; Guayaquil.

MARCOS, J. G., 1986 - Intercambio a Larga Distancia en América: El caso del Spondylus. In: Arqueología de la costa Ecuatoriana: nuevos enfoques (Marcos, J., ed.): 197-206; Guayaquil: Centro de Estudios Arqueológicos y Antropológicos y Corporación Nacional.

MARCOS, J. G., 1995 - El Mullo y el Pututo: la Articulación de la Ideología y el Tráfico a Larga Distancia en la formación del Estado Huancavilca . In: Primer Encuentro de Investigadores de la Costa Ecuatoriana en Europa (Álvarez, A., Álvarez, S., Fauria, C. \& Marcos, J. G., eds.): 97-142, Quito: Abya-Yala.

MORA, E., 1990 - Catálogo de Bivalvos Marinos del Ecuador. Boletín Científico y Técnico, X(1), 136 p.; Instituto Nacional de Pesca.

MASUCCI, M. A., 1995 - Marine shell Production and the Role of Domestic Craft Activities in the Economic of the Guangala Phase, Southwest Ecuador. Latin American Antiquity, 6 (1): 70-84; Washington D.C.

MASUCCI, M. A., 2000 - Informe de Prospecciones y Excavaciones Arqueológicas en el Valle de Chanduy, Cantón Santa Elena, Provincia del Guayas, 1998-1999; Guayaquil. Ms. en el Instituto Nacional de Patrimonio Cultural.

MESTER, A. M., 1990 - The Pearl Divers of Los Fraile: Archaeological and Ethnohistorical Explorations of Sumptuary Good Trade and Cosmology in the North and Central Andes. Unpublished Ph. D. Dissertation, Department of Anthropology, University of Illinois at Urban-Champaign.

MESTER, A. M., 1992 - Un taller Manteño de la concha de madreperla del sitio de Los Frailes (Manabí, Ecuador). In: 5000 Años de Ocupación: Parque Nacional Machalilla (Norton \& García, eds.): 41-49; Quito: Centro Cultural Ares-Editorial Abya-Yala.

MURRA, J. V., 1975 - El Tráfico del Mullu en la Costa del Pacífico. In: Formaciones Económicas y Políticas del Estado Inca (Murra, J. V., ed.): 255-267; Lima: IEP.

NORTON, P., 1986 - El Señorío de Salangone y la Liga de Mercaderes: el Cartel SpondylusBalsa. In: Arqueología y Etnohistoria del Sur de Colombia y Norte del Ecuador (Alcina \& Moreno, eds.): 131-143; Guayaquil: Museo del Banco Central del Ecuador. Miscelánea Antropológica Ecuatoriana 6.

NORTON, P., LUNNIS, R. \& NAILING, N., 1983 - Excavaciones en Salango, Provincia de Manabí, Ecuador, 9-72; Guayaquil: Museo del Banco Central del Ecuador. Miscelánea Antropológica Ecuatoriana 3.

PAULSEN, A. C., 1974 - The Thorny-Oyster and the Óbice of God: Spondylus and Strombus in Andean Prehistory. American Antiquity, 39 (4): 597-607; Washington D.C.: Society for American Archaeology.

STOTHERT, K. \& CARTER, B., 2000 - Informe Preliminar: resultados del rescate arqueológico en el Sitio M5 A3-362, Mar Bravo, Península de Santa Elena, Ecuador; Guayaquil. Ms. en el Instituto Nacional de Patrimonio Cultural.

VANHAEREN, M., 2003 - Speaking with Beads: the Evolucionary Significance of Beads making and use. Ms. presentado a From Tools to Symbols, From Early Hominids to Modern Humans International Round Table, Johannesburgo, 16-18 de Marzo. 
WIESSNER, P., 1982 - Risk, reciprocity and social influences on Kung San economics. Politics and History in Band Societies. In: Politics and history in band societies (Leacock, H. R. \& Lee, R. B., eds.): 61-84; London: Cambridge University Press.

ZEVAlLOS, C., 1995 - Nuestras Raíces Huancavilcas, 435 p.; Guayaquil: Casa de la Cultura Ecuatoriana Benjamín Carrión, Núcleo del Guayas. 